University of Wollongong

Research Online

Faculty of Engineering and Information

Faculty of Engineering and Information

Sciences - Papers: Part B

Sciences

2018

Dynamic interaction between grain boundary and stacking fault tetrahedron

\author{
Liang Zhang \\ University of Wollongong, Iz592@uowmail.edu.au \\ Cheng Lu \\ University of Wollongong, chenglu@uow.edu.au \\ Anh Kiet Tieu \\ University of Wollongong, ktieu@uow.edu.au \\ Yasushi Shibuta \\ University of Tokyo
}

Follow this and additional works at: https://ro.uow.edu.au/eispapers1

Part of the Engineering Commons, and the Science and Technology Studies Commons

Research Online is the open access institutional repository for the University of Wollongong. For further information contact the UOW Library: research-pubs@uow.edu.au 


\title{
Dynamic interaction between grain boundary and stacking fault tetrahedron
}

\author{
Abstract \\ We utilize molecular dynamics simulations to investigate the dynamic interaction between the grain \\ boundary (GB) and the stacking fault tetrahedron (SFT) in bicrystal copper. The grain boundary can \\ migrate itself under the shear strain and can serve as a sink to remove SFT. The sink efficiency of grain \\ boundaries is sensitive to their structural characteristics. The high-angle GBs can show a great ability to \\ remove SFT even at an extreme low temperature, while the increase of temperature can facilitate the \\ annihilation of SFT at the low-angle GBs. This study reveals a new possible GB-mediated damage healing \\ mechanism of irradiated materials. \\ Disciplines \\ Engineering | Science and Technology Studies \\ Publication Details \\ Zhang, L., Lu, C., Tieu, K. \& Shibuta, Y. (2018). Dynamic interaction between grain boundary and stacking \\ fault tetrahedron. Scripta Materialia, 144 78-83.
}




\title{
Dynamic interaction between grain boundary and stacking fault tetrahedron
}

\author{
Liang Zhang ${ }^{1,2, *}$, Cheng $\mathrm{Lu}^{1, *}$, Kiet Tieu ${ }^{1}$, Yasushi Shibuta ${ }^{2}$ \\ ${ }^{1}$ School of Mechanical, Materials and Mechatronic Engineering, University of Wollongong, Wollongong, NSW 2522, Australia \\ ${ }^{2}$ Department of Materials Engineering, University of Tokyo, Bunkyo-ku, Tokyo 113-8656, Japan \\ *Corresponding author. Tel.:+61416478011; fax:+61242213101; \\ E-mail: 1z592@uowmail.edu.au (L. Zhang); chenglu@uow.edu.au (C. Lu)
}

\begin{abstract}
We utilize molecular dynamics simulations to investigate the dynamic interaction between the grain boundary (GB) and the stacking fault tetrahedron (SFT) in bicrystal copper. The grain boundary can migrate itself under the shear strain and can serve as a sink to remove SFT. The sink efficiency of grain boundaries is sensitive to their structural characteristics. The high-angle GBs can show a great ability to remove SFT even at an extreme low temperature, while the increase of temperature can facilitate the annihilation of SFT at the low-angle GBs. This study reveals a new possible GB-mediated damage healing mechanism of irradiated materials.
\end{abstract}

Keywords: Molecular dynamics; Grain boundary; Vacancy; Dislocation

High-energy particles, such as neutron, proton, and ions, will develop point defects or defect clusters in materials under irradiation environment [1-3]. These defects may subsequently evolve into microstructural flaws which could deteriorate the physical properties of the irradiated materials and led to the direct structural failure $[4,5]$. Stacking fault tetrahedron (SFT) is a dominant type of vacancy cluster in various of irradiated face-centered cubic (fcc) metals due to its favorable structure that contains close-packed planes [6-9]. The structure of SFT is highly stable so that can act as a strong obstacle to the gliding dislocations, which results in hardening, embrittlement and plastic instabilities of materials [10,11]. The efficient removal of high-density SFTs is necessary to minimize void swelling and alleviate radiation hardening. However, the removal of SFT is very challenging and typically requires annealing at very high temperatures [12], incorporation of interstitials [13], or interaction with mobile dislocations [14].

It was reported that nanocrystalline materials had shown an improved radiation resistance compared to their coarser grained counterparts [15-18]. There are recently increasing interests on radiation tolerance of nanostructured materials because they possess a significant fraction of interfaces or grain boundaries (GBs) which can lead to the reduction of irradiation-induced defects [19-24]. The assumption that grain boundary can absorb point defects was confirmed by previous experimental observations. For examples, Chimi et al. [25] studied the effects of ion irradiation on nanocrystalline Au and showed that the irradiation-produced defects were thermally unstable because of the existence of a large volume fraction of GBs. Nita et al. [11] investigated the irradiation impact on the microstructure in nanostructured $\mathrm{Ni}$ and $\mathrm{Cu}$ specimens that were synthesized by electrodeposition and severe plastic deformation. They found that the irradiation-induced microstructure consists exclusively of SFTs, and the GB could be a major sink for the defects which resulted in a much lower density of SFTs than in the case of coarser grained material. The experimental findings suggest a possibility of the design and fabrication of radiation tolerant materials by reducing the grain size or via GB engineering. Meanwhile, the understanding of how defects interact with GB at the atomic scale is still in progressing [24, 26-28]. In particular, while GB can provide a large energetic driving force for self-interstitial atoms (SIAs) to aggregate on the GB, the radiation-induced vacancies and their clusters (e.g., voids and SFTs) usually show a less mobility than the SIAs [10, $13,22,29]$. In some irradiation experiments, the vacancy defects became nearly immobile under a low-temperature condition [25, 30,31]. Nevertheless, the annihilation of vacancies was still somehow enhanced by grain boundaries in these experiments, indicating the alternative GB-mediated mechanisms instead of the conventional vacancy diffusion mechanism that can assist the annihilation of vacancy defects within grain interiors. Atomistic simulations offered an opportunity to study the GB behaviors since the deformation conditions can be controlled and a detailed investigation of the underlying atomic scale processes can be made [32]. For example, by using molecular dynamics (MD) simulations, Bai et al. [13] proposed a 'recombination mechanism' at GB, i.e., the radiation-induced interstitials were firstly absorbed by the GB to form an interstitial-rich GB which can promote the vacancy-interstitial recombination subsequently. In this study, MD simulations were carried out to investigate the interaction between the shear-coupled GB motion and SFT in bicrystal $\mathrm{Cu}$. We found that the migrating GB can serve as a sink to remove SFT intragrain. Also, it was found that GB structure and temperature can have a significant influence on the sink efficiency.

The simulations were conducted using the parallel molecular dynamics code LAMMPS [33] with the embedded-atom method (EAM) potential for $\mathrm{Cu}$ [34]. The dimensions of each constructed bicrystal model were approximately $108 \AA \times 146 \AA \times 108 \AA(X \times Y \times Z)$, and the total number of atoms was about $1.5 \times 10^{5}$. The GB was obtained by rotating grain-A and grain-B along [001] tilt axis, and the SFT was placed in grain-A, as shown in Fig.1(a). Four symmetric tilt GBs were taken into consideration in this study; they are $\Sigma 5(310)\left(\theta=36.9^{\circ}\right) \mathrm{GB}, \Sigma 5(210)\left(\theta=53.1^{\circ}\right) \mathrm{GB}, \Sigma 37(750)$ $\left(\theta=71.1^{\circ}\right) \mathrm{GB}$, and $\Sigma 61(650)\left(\theta=79.6^{\circ}\right) \mathrm{GB}$. The four GBs were intentionally selected to represent both of the high-angle 
GB and the low-angle GB. Due to the symmetry of the fcc lattice, the misorientation angles from $0^{\circ}$ to $90^{\circ}$ covered all the distinct boundary structures of $<100>$ tilt GBs so that any GB that with the misorientation angle close to $0^{\circ}$ or $90^{\circ}$ was referred to the low-angle GB. Here, we tentatively defined the $\Sigma 37 \mathrm{~GB}$ and $\Sigma 61 \mathrm{~GB}$ as the low-angle GBs, while the two $\Sigma 5$ GBs were regarded as the high-angle ones. The detailed GB structures are shown in Fig.1(b) where all the GBs contain topologically identical structural units, as outlined by the red line. They differ only by the distance separating the structural units and by their positions relative to the GB plane, consisting either a flat $(\Sigma 5, \Sigma 61)$ or a zigzag ( $\Sigma 37)$ boundary plane. In particular, the structure of the two low-angle GBs can be described as an array of discrete perfect dislocations $(\mathbf{b}=1 / 2[\overline{1} 10])$, and the dislocation cores are formed by the structural units. The SFT was generated by introducing an equilateral triangular plate of vacancies in the (111) plane in grain-A. The triangular platelet contained 55 vacancies, 10 on each edge. After the energy minimization procedure, the vacancy plate evolved to an SFT followed the Silcox-Hirsch mechanism [35]. The size of the SFT is about $2.3 \mathrm{~nm}$ long on each edge, which is similar to the size of SFTs in copper observed in the experiment [36].

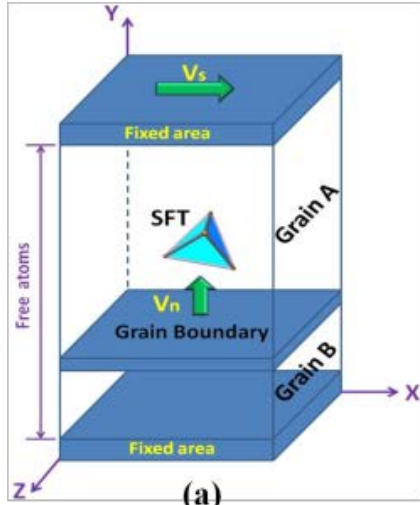

(a)

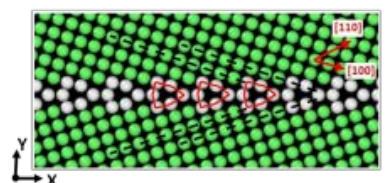

$\Sigma 5(310)\left(\theta=36.9^{\circ}\right) \mathrm{GB}$

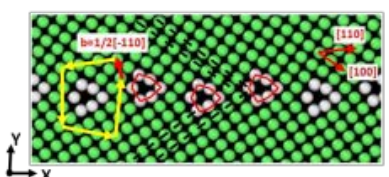

$\Sigma 37(750)\left(\theta=71.1^{\circ}\right) \mathrm{GB}$

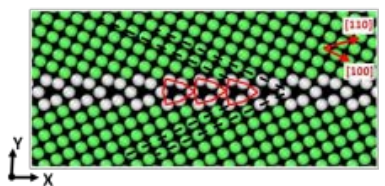

$\Sigma 5(210)\left(\theta=53.1^{\circ}\right) \mathrm{GB}$

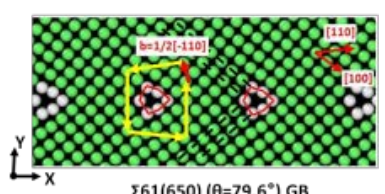

(b)

Figure 1. (a) Schematic of the simulation model. (b) Atomic images of the equilibrium structure of $\Sigma 5(310)\left(\theta=36.9^{\circ}\right) \mathrm{GB}, \Sigma 5(210)\left(\theta=53.1^{\circ}\right) \mathrm{GB}, \Sigma 37(750)$ $\left(\theta=71.1^{\circ}\right) \mathrm{GB}$, and $\Sigma 61(650)\left(\theta=79.6^{\circ}\right) \mathrm{GB}$. The GB structures are viewed along the [001] tilt axis and are colored according to the common neighbor analysis (CNA) parameter. The structural units are outlined by the red line. The Burgers vectors of the dislocations were determined by the standard Burgers circuit, as indicated by the yellow and red arrows

To simulate the coupled GB motion, we followed the previous method [37] to apply a shear deformation to the bicrystal model. The shear velocity was set as $\mathrm{V}_{\mathrm{s}}=2 \mathrm{~m} / \mathrm{s}$ throughout the simulation, and the time increment of simulations was fixed at $2 \mathrm{fs}$. By considering the effect of temperature, the simulations were carried out at $10 \mathrm{~K}, 300 \mathrm{~K}$ and $600 \mathrm{~K}$ respectively. Simulation results show that the GBs can show a normal motion $\left(\mathrm{V}_{\mathrm{n}}\right)$ under shear in all studied cases. Specifically, when the stress reached a critical level, the GB jumped upwards abruptly from its original place to another equilibrium position, accompanied by the release of local stress concentration [38]. The GB kept stationary until the energy barrier was overcome to reach the next local energy minimum as the shear strain progressed. This periodic process keeps driving the motion of the boundary and results in the GB migration upwards, as shown in the supplementary videos.

The migrating GB swept up the SFT in grain-A as it passed by, and some different interactions have been observed depending on the GB characteristics. Fig.2 shows the snapshots of interactions between GBs and the preexisting SFT at $10 \mathrm{~K}$. For $55(310) \mathrm{GB}$, the boundary plane moved upwards under shear and reached the SFT at about $0.7 \mathrm{~ns}$. The SFT annihilated gradually into the boundary plane as the GB migration progressed, and it was eventually absorbed by the GB, leaving behind a defect-free area, as shown in Fig.2(a). The same scenario was observed for $\Sigma 5(210)$ GB, where the SFT was completely removed by the migrating boundary plane, as shown in Fig.2(b). In the case of $\Sigma 37(750)$ GB, the SFT was destructed by the migrating GB at $10 \mathrm{~K}$, but the absorption of the SFT was incomplete. While the major part of the SFT structure was removed, the rest part broken down into the mono-vacancies and the small vacancy cluster, as shown in Fig.2(c). By checking the enlarged image, the total number of the remanent vacancies was counted as $n=7$. For

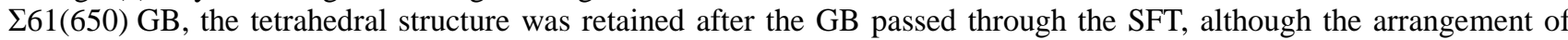
atoms at the edges of the SFT became less regular, as shown in Fig.2(d). The simulation results obtained at the extreme low temperature indicate that the GB structure can play a significant role in the removal of SFT. The high-angle GBs show a strong ability to annihilate the SFT, while the sink efficiency for absorbing SFT of the low-angle GBs is relatively weak or even not evidenced. This finding qualitatively agrees with the experimental results where the increased radiation tolerance was reported in the nanostructured materials which possess a large proportion of high-angle GBs [3]. On the other hand, the temperature in some irradiation experiments are so low (for instance, $100 \mathrm{~K}$ [25]) that the conventional vacancy diffusion mechanism is less possible functioned. The simulations conducted at $10 \mathrm{~K}$ in this study, in particular for the high-angle GBs, revealed a possible GB-mediated mechanism that can assist the removal of vacancy defects at low temperature. 

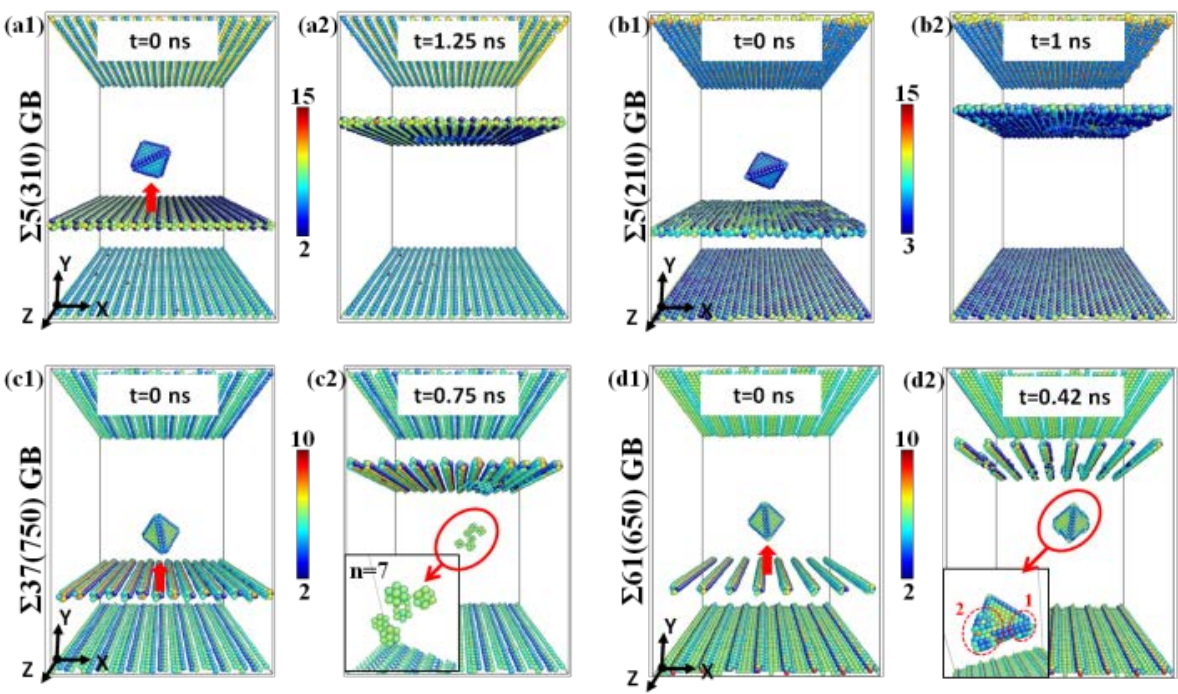

Figure 2. Snapshots of the atomic images before and after the interaction between SFT and different GBs at $10 \mathrm{~K}$. (a) $\Sigma 5$ (310) GB, (b) $\Sigma 5$ (210) GB, (c) $\Sigma 37$ (750) GB, and (d) 561(650) GB. The atoms are colored according to centrosymmetry value.

It is known that the structure of SFT consists of six stair-rod dislocations along the edges of the tetrahedron and the staking fault on the four $\{111\}$ planes [7]. On the other hand, the low-angle GB can be regarded as composed of an array of discrete dislocations. Therefore, the interaction between the low-angle GB and the SFT can be interpreted as a serial of dislocation activities. To further understand the low-angle GB-SFT interaction, the dislocation extraction algorithm [39, 40] was used to detect dislocation activities during the GB migration process, as shown in Fig.3.
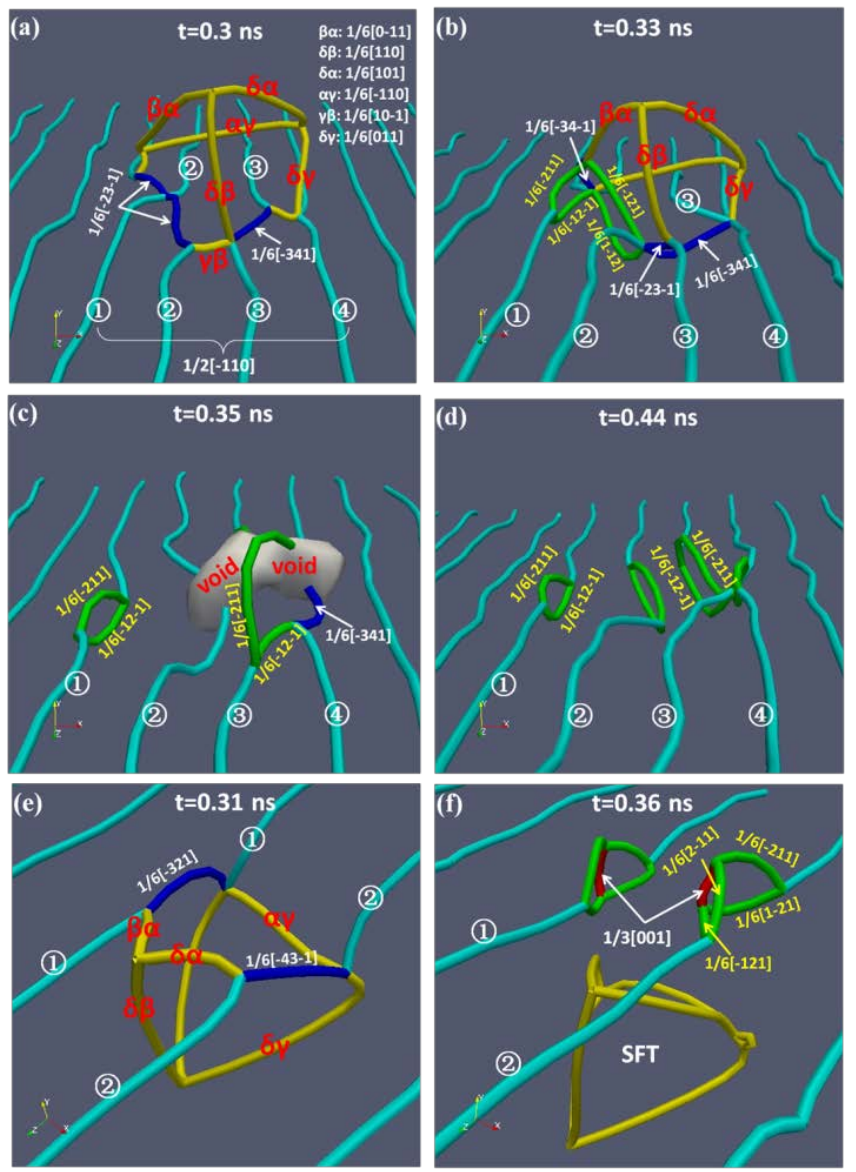

Figure 3. The extracted dislocation segments during the interactions between low-angle GBs and SFT at $10 \mathrm{~K}$. (a)-(d) $\Sigma 37(750)$ GB; (e)-(f) $\Sigma 61(650)$ GB. The extracted lines are colored according to the dislocation type. The light blue lines represent the perfect dislocations (PDs) at the GB, the yellow lines represent the stair-rod dislocations at the SFT, the green lines indicate the Shockley partial dislocations (SDs). The dark blue lines and the red line are the other types of dislocations. 
In the case of $\Sigma 37(750) \mathrm{GB}$, the migrating boundary reached SFT about 0.3 ns after the shear stress was applied. The GB dislocations with $\mathbf{b}=1 / 2[\overline{1} 10]$ reacted with the two stair-rod dislocations ( $\gamma \beta$ and $\delta \gamma$ ) on the two edges of SFT. The products of the reaction are the dislocations $\mathbf{b}=1 / 6[\overline{2} 3 \overline{1}]$ and $\mathbf{b}=1 / 6[\overline{3} 41]$, as indicated by the dark blue lines in Fig.3(a). There are four perfect dislocations (PDs) at the GB which interacted with the SFT, and they are indexed as 1 to 4 for illustration. At $\mathrm{t}=0.33 \mathrm{~ns}, \mathrm{PD}-1$ and PD-2 on the left side of SFT dissociated into two Shockley partial dislocations (SDs) respectively, which are colored by the green lines in Fig.3(b). Subsequently, the SD with $\mathbf{b}=1 / 6[121]$ slipped across the (11) plane of the SFT and removed the stacking fault on it. With the progress of the GB migration, the similar process has occurred on the right side of SFT where the SDs were dissociated from PD-3 and PD-4 and slipped across the SFT. Recall that, the SFT was constructed from a vacancy cluster plate. Thus, the result of dislocation activities was to split the complete tetrahedral structure into discrete mono-vacancies and small vacancy clusters. For example, Fig.3(c) shows that the SFT was destructed at $\mathrm{t}=0.35 \mathrm{~ns}$, and a void was formed during the interaction. While most of the vacancy defects can be absorbed by the boundary plane (i.e. PDs), some saturated ones were left in grain-B after the GB passed by (see Fig.2(c2)). The final configurations of the four PDs which participated in the interaction with SFT are shown in Fig.3(d). For each PD, a short segment of the dislocation line was dissociated into two SDs to accommodate the change of local GB structure after the absorption of vacancies from SFT. In the case of $\Sigma 61(650)$ GB, only two PDs at the boundary plane were involved in the interaction with SFT due to the larger interval distance between the neighboring dislocations, as shown in Fig.3(e). While the four PDs at $\Sigma 37(750)$ GB can result in the destruction of SFT, the two PDs at $\Sigma 61(650)$ GB played little role in the removal of SFT. The lost vacancies from SFT into the two PDs did not change the tetrahedral structure during the interaction, as indicated by the network of the stair-rod dislocations in Fig.3(f). Each dislocation line of the low-angle GBs is corresponding to a single column of structural units along the $\mathrm{Z}$ axis. Therefore, the different results in Fig.3 may largely depend on the free volume that associated with the structural units at the boundary plane. For the high-angle GBs, the high-density structural units can provide large free volume to accommodate the vacancies that dissociated from the SFT. Also, the high-density structural units can provide continuous diffusion path along the boundary plane when GB was sliding under shear strain, which helps to release the local vacancies concentration at boundary plane and improves its sink ability accordingly. The dislocation analysis results in return highlight the important role of the GB structure on the removal of SFT. The heterogeneous GB diffusion and GB sliding were considered in the previous numerical simulation studies, and they were found to account for the recoverable creep deformation [41, 42] and the enhanced rate-sensitivity [43] in nanostructured materials.

The influence of temperature on the GB sink efficiency for removing SFT was investigated at $300 \mathrm{~K}$ and $600 \mathrm{~K}$. As expected, the complete annihilation of SFT was observed again for the $\Sigma 5(310)$ GB and $\Sigma 5(210)$ GB at higher temperature. Fig.4 shows the snapshots of interactions between the two low-angle GBs and the SFT at $300 \mathrm{~K}$ and $600 \mathrm{~K}$. Similar to the simulation case at $10 \mathrm{~K}$, the incomplete annihilation of SFT was observed in the case of $\Sigma 37(750)$ GB, leaving the scattered vacancies behind, as shown in the enlarged atomic images in Fig.4(a) and Fig.4(b). The remanent vacancies were counted as $n=6$ and $n=3$ at $300 \mathrm{~K}$ and $600 \mathrm{~K}$ respectively. For $\Sigma 61(650) \mathrm{GB}$, the increase of temperature to $300 \mathrm{~K}$ did not essentially improve its sink efficiency, the complete SFT still located at its original place when the GB has passed by, as shown in Fig.4(c). However, the SFT no longer existed when the temperature has increased to $600 \mathrm{~K}$. The main body of the SFT has been removed by the migrating GB, and only two mono-vacancies were left behind, as shown in Fig.4(d). The dynamic process of interaction in Fig.4 was presented in the supplementary videos. The simulations performed at $300 \mathrm{~K}$ and $600 \mathrm{~K}$ demonstrate the role of temperature in improving the ability to absorb SFT for the low-angle GBs, and the results confirmed the general assumption that the GB could exhibit higher sink efficiency at increased temperature. The previous atomistic studies have shown that the energy barriers for defect migration are lower at GB than in the bulk [13, 22]. Thus, diffusive transfer along the grain boundaries (GB diffusion) occurs much faster than in their bulk (bulk diffusion). Also, the increased temperature can facilitate the vacancies to overcome the activation energy barrier at GB due to the improved atomic thermal motion. Therefore, the SFT which usually has a stable structure within grain interiors became dissociated at GB when the temperature has increased; this is the case for both high-angle and low-angle GBs in this study.

Moreover, after the energy minimization procedure at $0 \mathrm{~K}$, the boundary energy was calculated as $925 \mathrm{~mJ} \mathrm{~m}^{-2}$ for $\Sigma 5(310) \mathrm{GB}, 955 \mathrm{~mJ} \mathrm{~m}^{-2}$ for $\Sigma 5(210) \mathrm{GB}, 742 \mathrm{~mJ} \mathrm{~m}^{-2}$ for $\Sigma 37(750) \mathrm{GB}$, and $549 \mathrm{~mJ} \mathrm{~m}^{-2}$ for $\Sigma 61(650)$ GB. From the perspective of energy, the GB with higher boundary energy may act as a stronger sink of defects and thus lead to its enhanced absorbing ability for the SFT. This point was confirmed by the GB-SFT interaction at low temperature, where the sink efficiency of the GB ( $\left.\eta_{\mathrm{GB}}\right)$ at $10 \mathrm{~K}$ is $\eta_{(310)}=\eta_{(210)}>\eta_{(750)}>\eta_{(650)}$. The elevated temperature increased the GB energy and can improve its sink ability correspondingly. Therefore, the result of GB-SFT interaction at $600 \mathrm{~K}$ is $\eta_{(310)}=\eta_{(210)} \approx \eta_{(750)} \approx \eta_{(650)}$. It was recently reported that the nanotwinned metals had shown an enhanced radiation tolerance with a high density of coherent twin boundaries (CTBs), and a large number of radiation-induced SFTs can be removed during their interactions with abundant CTBs [44-46]. The experimental evidence renewed the conventional understanding that CTB was a less effective defect sink due to its simple boundary structure and its extreme low boundary energy (calculated as $26 \mathrm{~mJ} \mathrm{~m}^{-2}$ [47]). The improved sink ability of the CTB may largely depend on its incorporation with the incoherent twin boundaries (ITBs) and the interstitial atoms. Specifically, the aggregation and transportation of interstitial atoms at CTB can change its local structure to become a higher energy segment which can subsequently be a trap of the vacancy defect clusters (e.g., SFT) [45]. In addition, abundant ITBs were generated along CTBs, and the frequent migration of ITBs was observed under the experimental conditions. The ITBs are effective defect sinks that can accommodate radiation induced defects as the regular high-angle GBs do [21, 46]. These factors were not 
considered when the biased sink ability of CTB was reported in the previous simulation works where only the perfect CTB structure was studied [28, 48, 49]. Therefore, it is inspired to conduct a further simulation study by constructing a comprehensive model with both CTB and other types of crystal defects.
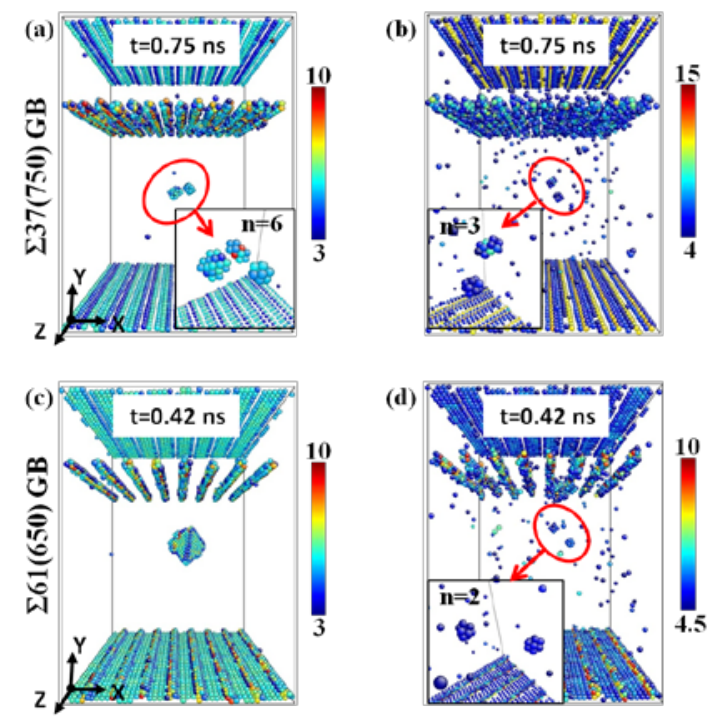

Figure 4. Snapshots of the atomic images after the GB-SFT interaction. (a) $\Sigma 37(750)$ GB at $300 \mathrm{~K}$, (b) $\Sigma 37(750)$ GB at $600 \mathrm{~K}$, (c) $\Sigma 61(650) \mathrm{GB}$ at $300 \mathrm{~K}$, and (d) $\Sigma 61(650) \mathrm{GB}$ at $600 \mathrm{~K}$. The atoms are colored according to centrosymmetry value.

In summary, the simulation results indicate that the migrating GB can serve as a sink to remove SFT. Although only the limited cases were considered in this study, the result highlights the major variations in the sink efficiency of absorbing SFT by different GBs. The high-angle GBs showed a great ability to remove SFT even at the extreme low temperature, while the sink efficiency of the low-angle GBs was enhanced significantly from $10 \mathrm{~K}$ to $600 \mathrm{~K}$. The simulation result implies a new possible self-healing mechanism of the irradiated materials and can provide useful information for the design of radiation tolerant materials.

This work was supported by Australian Research Council Discovery Project (DP170103092). Access to the high performance computer (HPC) facilities provided by the University of Wollongong is also acknowledged. Liang Zhang would like to acknowledge the Postdoctoral Fellowship Program (P17711) from Japan Society for the Promotion of Science (JSPS).

\section{References}

[1] P.J. Maziasz, J. Nucl.Mater. 205 (1993) 118-145.

[2] B.N. Singh, S.J. Zinkle, J. Nucl.Mater. 206 (1993) 212-229.

[3] R.W. Grimes, R.J.M. Konings, L. Edwards, Nature Mater. 7 (2008) 683-685.

[4] B.D. Wirth, Science 318 (2007) 923-924.

[5] G. Ackland, Science 327 (2010) 1587-1588.

[6] M. Kiritani, Mater. Chem. Phys. 50 (1997) 133-138.

[7] M.H. Loretto, P.J. Phillips, M.J. Mills, Scr. Mater. 94 (2015) 1-4.

[8] L. Zhang, C. Lu, G. Michal, G. Deng, K. Tieu, Scr. Mater. 136 (2017) 78-82.

[9] D.S. Aidhy, C. Lu, K. Jin, H. Bei, Y. Zhang, L. Wang, W.J. Weber, Scr. Mater. 114 (2016) 137-141.

[10] E. Martínez, B.P. Uberuaga, Sci. Rep. 5 (2015) 9084.

[11] N. Nita, R. Schaeublin, M. Victoria, J. Nucl. Mater. 329-333 (2004) 953-957.

[12] B.N. Singh, S.I. Golubov, H. Trinkaus, D.J. Edwards, M. Eldrup, J. Nucl. Mater. 328 (2004) 77-87.

[13] X.M. Bai, A.F. Voter, R.G. Hoagland, M. Nastasi, B.P. Uberuaga, Science 327 (2010) 1631-1634.

[14] J.S. Robach, I.M. Robertson, H.J. Lee, B.D. Wirth, Acta Mater. 54 (2006) 1679-1690.

[15] M.J. Demkowicz, R.G. Hoagland, J.P. Hirth, Phys. Rev. Lett. 100 (2008).

[16] A. Kilmametov, A. Balogh, M. Ghafari, C. Gammer, C. Mangler, C. Rentenberger, R. Valiev, H. Hahn, Radiat. Eff. Defects Solids, 167 (2012) 631-639.

[17] K.Y. Yu, Y. Liu, C. Sun, H. Wang, L. Shao, E.G. Fu, X. Zhang, J. Nucl. Mater. 425 (2012) 140-146.

[18] I.J. Beyerlein, N.A. Mara, J.S. Carpenter, T. Nizolek, W.M. Mook, T.A. Wynn, R.J. McCabe, J.R. Mayeur, K. Kang, S. Zheng, J. Wang, T.M. Pollock, J. Mater. Res. 28 (2013) 1799-1812.

[19] F.J. Pérez Pérez, R. Smith, Nucl. Instrum. Methods Phys. Res., Sect.B 153 (1999) 136-141.

[20] F. Gao, H.L. Heinisch, R.J. Kurtz, J. Nucl. Mater. 386-388 (2009) 390-394.

[21] W.Z. Han, M.J. Demkowicz, E.G. Fu, Y.Q. Wang, A. Misra, Acta Mater. 60 (2012) 6341-6351.

[22] M.A. Tschopp, K.N. Solanki, F. Gao, X. Sun, M.A. Khaleel, M.F. Horstemeyer, Phys. Rev. B 85 (2012) 064108.

[23] W. Han, M.J. Demkowicz, N.A. Mara, E. Fu, S. Sinha, A.D. Rollett, Y. Wang, J.S. Carpenter, I.J. Beyerlein, A. Misra, Adv. Mater. 25 (2013) 
6975-6979.

[24] X.M. Bai, B.P. Uberuaga, JOM, 65 (2013) 360-373.

[25] Y. Chimi, A. Iwase, N. Ishikawa, M. Kobiyama, T. Inami, S. Okuda, J. Nucl. Mater. 297 (2001) 355-357.

[26] I.J. Beyerlein, M.J. Demkowicz, A. Misra, B.P. Uberuaga, Prog. Mater. Sci. 74 (2015) 125-210.

[27] V. Borovikov, X.Z. Tang, D. Perez, X.M. Bai, B.P. Uberuaga, A.F. Voter, Nucl. Fusion 53 (2013) 063001.

[28] E. Martínez, B.P. Uberuaga, I.J. Beyerlein, Phys. Rev. B 93 (2016) 054105

[29] X. Li, W. Liu, Y. Xu, C.S. Liu, B.C. Pan, Y. Liang, Q.F. Fang, J.L. Chen, G.N. Luo, G.H. Lu, Z. Wang, Acta Mater. 109 (2016) 115-127. [30] M. Rose, A.G. Balogh, H. Hahn, Nucl. Instrum. Methods Phys. Res., Sect.B 127-128 (1997) 119-122.

[31] T.D. Shen, S. Feng, M. Tang, J.A. Valdez, Y. Wang, K.E. Sickafus, Appl. Phys. Lett. 90 (2007) 263115.

[32] L. Zhang, C. Lu, K. Tieu, Comp. Mater. Sci. 118 (2016) 180-191. [33] S. Plimpton, J. Comp. Phys. 117 (1995) 1-19.

[34] Y. Mishin, M.J. Mehl, D.A. Papaconstantopoulos, A.F. Voter, J.D. Kress, Phys. Rev. B 63 (2001) 2241061-22410616.

[35] L. Zhang, C. Lu, K. Tieu, L. Su, X. Zhao, L. Pei, Mater. Sci. Eng. A, 680 (2017) 27-38.

[36] Y. Dai, M. Victoria, Acta Mater. 45 (1997) 3495-3501.

[37] L. Zhang, C. Lu, K. Tieu, X. Zhao, L. Pei, Nanoscale, 7 (2015) 7224-7233.

[38] L. Zhang, C. Lu, G. Michal, K. Tieu, X. Zhao, G. Deng, Phys. Status Solidi (b), 254 (2017) 1600477.

[39] A. Stukowski, Modell. Simul. Mater. Sci. Eng. 20 (2012) 045021.

[40] A. Stukowski, V.V. Bulatov, A. Arsenlis, Modell. Simul. Mater. Sci. Eng. 20 (2012) 085007.

[41] Y.J. Wei, A.F. Bower, H.J. Gao, Scr. Mater. 57 (2007) 933-936.

[42] Y.J. Wei, A.F. Bower, H.J. Gao, J. Mech. Phys. Solids, 56 (2008) 1460-1483.

[43] Y.J. Wei, A.F. Bower, H.J. Gao, Acta Mater. 56 (2008) 1741-1752.

[44] K.Y. Yu, D. Bufford, C. Sun, Y. Liu, H. Wang, M.A. Kirk, M. Li, X. Zhang, Nature Comm. 4 (2013).

[45] J. Li, K.Y. Yu, Y. Chen, M. Song, H. Wang, M.A. Kirk, M. Li, X. Zhang, Nano Lett. 15 (2015) 2922-2927.

[46] Y. Chen, J. Li, K.Y. Yu, H. Wang, M.A. Kirk, M. Li, X. Zhang, Acta Mater. 111 (2016) 148-156.

[47] L. Zhang, C. Lu, K. Tieu, L. Pei, X. Zhao, K. Cheng, Mater. Res. Express, 2 (2015) 035009.

[48] M.J. Demkowicz, O. Anderoglu, X. Zhang, A. Misra, J. Mater. Res. 26 (2011) 1666-1675.

[49] X.M. Bai, L.J. Vernon, R.G. Hoagland, A.F. Voter, M. Nastasi, B.P. Uberuaga, Phys. Rev. B 85 (2012). 